\title{
Considerações à anestesia geral de cães e gatos com oftalmopatias
}

\author{
Considerations for general anesthesia in dogs and cats with eye diseases \\ Consideraciones para la anestesia general de perros y gatos con oftalmopatías
}

Recebido: 22/06/2021 | Revisado: 30/06/2021 | Aceito: 04/07/2021 | Publicado: 15/07/2021

Carolina Araújo Neves
ORCID: https://orcid.org/0000-0002-0666-6327
Universidade Federal de Jataí, Brasil
E-mail: acaroaraujo@ gmail.com
Laurenzo Vicentini Pais Mendonça
ORCID: https://orcid.org/0000-0002-4929-9037
Universidade Federal de Jataí, Brasil
E-mail: laurenzovpm@ gmail.com
Mariana Ferreira da Silva
ORCID: https://orcid.org/0000-0001-5114-431X
Universidade Federal de Jataí, Brasil
E-mail: mariana.ferreira@ discente.ufg.br
João Marcelo Carvalho do Carmo
ORCID: https://orcid.org/0000-0002-6331-992X
Universidade Federal de Jataí, Brasil
E-mail: Joaomarcelo_c2@ @otmail.com
Nathany Arcaten
Hathe
ORCID: https://orcid.org/0000-0001-9752-8095
Universidade Federal de Jataí, Brasil
E-mail: nathanyarcaten@ discente.ufg.br
Klaus Casaro Saturnino
ORCID: https://orcid.org/0000-0001-8493-8669
Universidade Federal de Jataí, Brasil
E-mail: klaus.sat@ ufg.br
Andréia Vitor Couto do Amaral
ORCID: https://orcid.org/0000-0001-6406-2372
Universidade Federal de Jataí, Brasil
E-mail: andreiavcvet@ufg.br

\section{Resumo}

O objetivo do presente trabalho é apresentar uma rápida e prática revisão de literatura sobre as particularidades da anestesia geral para cães e gatos com oftalmopatias, fazendo um breve relato das principais considerações que o anestesista deverá ter em vista, frente ao paciente com alteração ocular. Frequentemente são utilizados barbitúricos, propofol, agonistas dos receptores $\alpha 2$-adrenérgicos, benzodiazepínicos, fenotiazinas e opioides em protocolos para anestesia geral de procedimentos cirúrgicos oftálmicos em cães e gatos, podendo promover alterações na pressão intraocular, na produção lacrimal, no tamanho da pupila, na centralização do olho, nos reflexos palpebral, corneal e oculocardíaco. A elaboração de um protocolo anestésico para determinado paciente envolve não apenas a escolha apropriada do agente anestésico, mas também um plano de conduta capaz de assegurar a qualidade do resultado pósoperatório. Isto requer conhecimento do estado físico do paciente e do procedimento oftálmico específico a ser realizado, além de familiaridade com a fisiologia e farmacologia ocular.

Palavras-chave: Analgesia; Anestésicos; Olho.

\begin{abstract}
The aim of this paper is to present a quick and practical review of the literature on the particularities of general anesthesia for dogs and cats with ophthalmopathies, making a brief report of the main considerations that the anesthesiologist should have in view of patients with ocular changes. Barbiturates, propofol, $\alpha 2$-adrenergic receptor agonists, benzodiazepines, phenothiazines and opioids are often used in protocols for general anesthesia of ophthalmic surgical procedures in dogs and cats, which may promote changes in intraocular pressure, tear production, pupil size, in eye centering, in the eyelid, corneal and oculocardiac reflexes. The elaboration of an anesthetic protocol for a specific patient involves not only the appropriate choice of anesthetic agent, but also a management plan capable of assuring the quality of the postoperative result. This requires knowledge of the patient's physical condition and the specific ophthalmic procedure to be performed, as well as familiarity with ocular physiology and pharmacology.
\end{abstract}

Keywords: Analgesia, Anesthetics; Eye. 


\begin{abstract}
Resumen
El objetivo de este trabajo es presentar una revisión rápida y práctica de la literatura sobre las particularidades de la anestesia general para perros y gatos con oftalmopatías, haciendo un breve informe de las principales consideraciones que debe tener el anestesiólogo ante los pacientes con alteraciones oculares. Los barbitúricos, propofol, agonistas del receptor $\alpha 2$-adrenérgico, benzodiazepinas, fenotiazinas y opioides se utilizan a menudo en protocolos de anestesia general de procedimientos quirúrgicos oftálmicos en perros y gatos, que pueden promover cambios en la presión intraocular, producción de lágrimas, tamaño de la pupila, centrado de ojos en los reflejos palpebral, corneal y oculocardíaco. La elaboración de un protocolo anestésico para un paciente específico implica no solo la elección adecuada del agente anestésico, sino también un plan de manejo capaz de asegurar la calidad del resultado postoperatorio. Esto requiere el conocimiento de la condición física del paciente y el procedimiento oftálmico específico a realizar, así como familiaridad con la fisiología y farmacología ocular.
\end{abstract}

Palabras clave: Analgesia; Anestésicos; Ojo.

\title{
1. Introdução
}

A rotina da anestesia de cães e gatos engloba, não só, mas também, pacientes com oftalmopatias, que possuem considerações específicas. A seleção de um protocolo anestésico para cirurgia oftalmológica deverá considerar os efeitos sobre a pressão intraocular (PIO), o tamanho da pupila, a posição do bulbo ocular, a produção de lágrimas e a possibilidade de indução do reflexo oculocardíaco (ROC) durante a manipulação cirúrgica do bulbo ocular (Gross \& Giuliano, 2017).

Frequentemente, os desafios na anestesia em oftalmologia de cães e gatos incluem a escolha de fármacos que não promova aumentos na pressão intraocular; que assegure que o paciente não tenha movimentos súbitos no globo ocular, cabeça ou mesmo qualquer parte do corpo durante a cirurgia; mantenha o globo ocular e pupila centralizados e que possa garantir uma recuperação pós-operatória tranquila e sem dor, abolindo qualquer auto traumatismo ocular (Madruga et al., 2015; Gross \& Pablo, 2015). Alguns desses objetivos podem parecer conflitantes, como por exemplo, ao assegurar que não haja risco de movimento súbito do paciente durante a cirurgia, quer seja um sutil reflexo corneal ou palpebral, pode fazer com que o anestesista mantenha um plano anestésico excessivamente profundo, deprimindo as funções cardiovasculares e respiratórias (Gross \& Pablo, 2015).

Assim, cada fármaco deverá ter seu efeito reconhecido nas principais funções oculares que se deseja monitorar, de forma a escolher o protocolo ideal (Madruga et al., 2015). O uso de bloqueios locais também deverá ser considerado pelo anestesista (Pinto et al., 2021), entretanto não constitui objeto desta revisão.

O objetivo do presente trabalho é apresentar uma rápida e prática revisão de literatura sobre as particularidades da anestesia geral para cães e gatos com oftalmopatias, fazendo um breve relato das principais considerações que o anestesista deverá ter em vista, frente ao paciente com alteração ocular.

\section{Metodologia}

Realizou-se uma revisão narrativa não sistematizada, utilizando artigos científicos, monografias, teses e dissertações publicadas e disponíveis em bases de dados online: Periódico Capes (Coordenação de Aperfeiçoamento de Pessoal de Nível Superior), SciELO (Scientific Electronic Library Online) e Google Acadêmico, além de livros específicos do tema. Conforme metodologia citada por Borges e colaboradores (2020), foram excluídos os estudos que não abordavam a temática principal em análise, bem como artigos opinativos que não estavam apoiados em dados de pesquisa.

A pesquisa bibliográfica se desenvolveu para explicar as particularidades da anestesia geral para cães e gatos com oftalmopatias. Foi utilizando o conhecimento disponível a partir das teorias publicadas em livros ou artigos para auxiliar na compreensão, atualização ou objeto de revisão para pesquisas futuras, assim como proposto por Koche (2011). 


\section{Revisão de Literatura}

\section{Considerações anatomofisiológicas oculares}

É necessário conhecer algumas características anatomofisiológicas do olho de relevância para anestesia ocular. Apesar das diferenças entre raças e espécies de cães e gatos, a anatomia e fisiologia do olho são bem semelhantes, podendo dividi-lo em três camadas ou túnicas: a fibrosa ou esclerótica, a vascular ou uveal e a nervosa ou retina (Samuelson, 2013; Esson, 2015). A esclera é a camada mais externa da qual faz parte a córnea. A camada vascular, também chamada de trato uveal, é composta pela íris, corpo ciliar e coróide, sendo responsável pela nutrição do globo ocular, produção de aquoso (corpo ciliar) e controle da entrada de luz para a retina por meio da fenda pupilar (Maggs, 2013; Murphy et al., 2012; Esson, 2015). Neste sentido é importante ressaltar que o sistema nervoso simpático é responsável pela dilatação da pupila, por meio da contração dos músculos radiais da íris e o parassimpático pelo fechamento da fenda pupilar, pela contração do músculo esfíncter da íris (Ofri, 2013a). O reflexo fotopupilar possui estimulação parassimpática, é mediado pelo nervo Óptico em sua via aferente e pelo nervo Oculomotor em sua via referente e é bastante utilizado na anestesiologia. Já as fibras simpáticas originam-se no plexo carotídeo e fazem um percurso mais longo, passam no gânglio ciliar, e inervam o músculo radial da íris (Ofri, 2013a; Samuelson, 2013).

A área entre o limbo da córnea e a retina é chamada de Pars plana. Neste ponto não há camada de retina, é um local de entrada seguro para injeções intravítreas e procedimentos de vitrectomia (Murphy et al., 2012). A câmara vítrea é preenchida por vítreo e ocupa grande proporção do globo ocular, sendo que, sua tração poderá ser responsável por descolamento de retina ou por luxações da lente (Ofri, 2013b). A retina é nutrida pela coróide, e derrames sanguíneos neste segmento vascular causam descolamento de retina e perda da visão (Ofri, 2013c).

Externamente, os músculos extraoculares são responsáveis por mover o Globo ocular. São sete músculos que surgem de um anel fibroso próximo ao ápice do espaço orbital e se inserem na esclera, formando assim um cone. No espaço intraconal, atrás do bulbo ocular, estão o nervo óptico, a artéria e a veia oftálmica (Samuelson, 2013)

As pálpebras possuem a função de proteção do globo ocular, produção de lágrima e recebem inervação do Facial e do Trigêmeo (Miller, 2013). A terceira pálpebra é inervada pelo Oculomotor, e se movimenta cobrindo porção da superfície ocular com a retração do bulbo ocular (Ofri, 2013a).

\section{Pressão intraocular}

Durante a anestesia de procedimentos oftálmicos é de suma importância a realização de uma intubação endotraqueal o mais suave possível, minimizando a estimulação laríngea, visto que é a etapa que tem mais chances de ter um aumento da pressão intraocular (PIO) significativamente (pelo menos 10 a $20 \mathrm{mmHg}$ ) (Donlon, 1988).

A diminuição da PIO, induzida pela maioria dos anestésicos, pode ser determinada em decorrência de uma combinação de fatores, incluindo depressão dos centros diencefálicos que regulam a PIO, aumento do fluxo do aquoso no ângulo iridocorneal, diminuição das pressões sanguíneas venosa e arterial e relaxamento da musculatura extraocular (Donlon, 2000).

O uso da cetamina isoladamente é contraindicado em pacientes com glaucoma, elevação da PIO ou potencial ruptura do bulbo ocular, pois provoca contração dos músculos extraoculares, o que pode resultar em elevação da PIO (Jia, Cepurna \& Johnson, 2000).

\section{Reflexo oculocardíaco}

O reflexo oculocardíaco (ROC) é um reflexo trigeminovagal (nervos cranianos $\mathrm{V}$ e $\mathrm{X}$ ), que pode ser induzido por pressão ou tração do bulbo ocular, traumatismo ou dor ocular, pressão exercida por massa ou hematoma orbital, por bloqueio 
retrobulbar ou peribulbar. O início do ROC manifesta-se na forma de arritmias cardíacas, que podem incluir bradicardia, ritmos nodais, batimentos ectópicos, fibrilação ventricular ou assistolia (Donlon, 2000).

O ROC foi considerado de importância clínica menor em cães submetidos à cirurgia oftálmica quando a técnica anestésica utilizada produz inconsciência adequada, relaxamento muscular satisfatório e hipocapnia leve (Clutton, Boyd \& Richards, 1988).

\section{Tamanho da pupila}

O tamanho da pupila é de suma importância na facectomia e em cirurgias intraoculares que exigem que a pupila esteja em total midríase, embora a maioria dos anestésicos gerais induz miose (Gross \& Giuliano, 2007). Dessa forma, a administração de midriáticos, como a epinefrina e fenilefrina, agentes simpatomiméticos, e parasimpatolíticos, como a tropicamida e atropina, são utilizadas topicamente, em forma de colírios, para promoção de midríase para procedimentos cirúrgicos. O uso de anti-inflamatórios não esteroidais (AINES) contribui para impedir a ocorrência de miose e podem ser usados durante a cirurgia intraocular, uma vez que prostaglandinas e outros mediadores da inflamação podem exercer um efeito direto sobre o músculo constritor da íris. Já em casos que seja requerida miose, poderá ser utilizada a pilocarpina colírio (Kaswan, Quandt \& Moore, 1992; Sharpe \& Pickworth, 1985).

\section{Posição do bulbo ocular}

Durante a anestesia geral, poderá ocorrer a movimentação do bulbo ocular, e a sua posição pode variar entre as espécies e de acordo com os planos de anestesia. A paralisia com bloqueadores neuromusculares ou anestesia regional retrobulbar com anestésicos locais durante a anestesia geral deve eliminar os reflexos oculares e possibilitar o posicionamento do bulbo ocular, sem a necessidade de tração manual, reduzindo, assim, a possibilidade de expulsão do conteúdo do bulbo ocular ou o desencadeamento do ROC (Clutton et al., 1988).

Ribeiro e colaboradores (2009) estudaram o efeito do propofol na posição do bulbo ocular e propuseram cinco planos anestésicos, observando a centralização do globo ocular no quarto e no quinto plano. Assim, observa-se que o posicionamento central do olho com o uso do propofol requer a manutenção de planos anestésicos profundos. Por esse motivo, a associação com drogas miorrelaxantes e bloqueios locorregionais são amplamente utilizados na rotina da anestesia geral oftálmica.

\section{Produção de lágrimas}

Foi demonstrada a ocorrência de diminuição na produção de lágrima em cães durante a anestesia geral, atribuindo-se à depressão das vias autônomas, sugerido assim, a lubrificação da superfície ocular a cada 90 minutos durante o procedimento (Krupin, Cross \& Becker, 1977; Shepard, Accola \& Lopez, 2011). De acordo com Grubb (2020), um lubrificante deve ser aplicado após a indução para proteger os olhos, seguido de sua reaplicação periódica, sugerindo a cada duas horas.

\section{Reflexos corneal e palpebral}

Durante os procedimentos cirúrgicos oftálmicos é fundamental a manutenção da ausência dos reflexos corneal e palpebral. Ambos reflexos possuem como via aferente fibras sensitivas do nervo Trigêmeo, que, ao estímulo da córnea ou das pálpebras, possuem como resposta o fechamento das pálpebras, mediado por fibras motoras eferentes do nervo Facial (Ofri, 2013a). Considerações sobre a associação de técnicas de bloqueio local para cirurgias oculares em cães e gatos estão disponíveis (Pinto et al., 2021).

Ribeiro e colaboradores (2009) observaram o efeito do propofol nos reflexos palpebral e corneal durante a indução anestésica em cães, identificando cinco planos anestésicos diferentes. A profundidade para intubação orotraqueal era vista no 
quinto plano, com ausência de reflexo palpebral, globo ocular centrado, ausência de reflexo corneano (Ribeiro et al., 2009). Isso nos mostra que a manutenção de ausência de reflexos oculares ideal para cirurgia oftálmica requer anestesia geral em planos profundos ou a associação de técnicas, evidenciando, mais uma vez, o uso de relaxantes neuromusculares e de bloqueios locorregionais em conjunto.

É primordial monitorar os reflexos palpebral e corneal também durante o retorno anestésico, atentando-se à necessidade de aplicar pomada ou gel lubrificante para os olhos a cada duas horas durante o período de recuperação, até que o reflexo de piscar adequado esteja presente no paciente (Grubb et al., 2020).

\section{Sedativos e anestésicos injetáveis}

Barbitúricos e propofol

O propofol é amplamente utilizado para indução anestésica intravenosa e intubação orotraqueal durante procedimentos cirúrgicos oftálmicos. De acordo com Batista e colaboradores, (2000), cães submetidos a infusão intravenosa contínua de propofol não apresentaram alterações significativas da PIO quando comparados aos seus valores basais. Em comparação com o tiopental, Echeverría-Varela \& Pineda-Mendoza (2007) relataram que logo após a intubação, houve uma maior eficácia por parte do propofol em conservar a estabilidade da PIO. Já Webb e colaboradores (2018) observaram que o propofol aumentou discretamente a PIO em cães sem glaucoma, mas manteve a PIO estável durante a cirurgia de em cães glaucomatosos.

Pontes e colaboradores (2010) compararam a produção lacrimal durante a anestesia feitos da indução com o tiopental ou com o propofol e observaram que ambas as drogas levaram à diminuição considerável na produção lacrimal, sugerindo a proteção da córnea e conjuntiva do paciente durante a anestesia.

\section{Agonistas dos receptores $\alpha 2$-adrenérgicos}

A xilazina diminui a PIO ao produzir depressão da função simpática e diminuição da produção de humor aquoso (Burke \& Potter, 1986). Além disso, provoca midríase em algumas espécies e a aplicação tópica produz catarata no olho que for instilado a mesma, enquanto o olho contralateral permanece normal (Hsu, Lee \& Betts, 1981; Calderone, Grimes \& Shalev, 1986).

A administração tópica de medetomidina diminui a PIO, enquanto produz midríase, sugerindo que existem receptores a2-adrenérgicos nos olhos que estão envolvidos na regulação da PIO (Jin, Wilson \& Elko, 1991; Potter \& Ogidigben, 1991). Já a administração por via intravenosa em cães resultou em miose, sem diminuição significativa da PIO (Verbruggen et al., 2000).

A administração intramuscular tanto da dexmedetomidina quanto da medetomidina diminuiu o diâmetro pupilar de cães quando associadas com a acepromazina (Aghababaei et al., 2021). Em estudo realizado por Aghababaei e colaboradores (2021) foi observado a diminuição da produção de lágrima com o uso da medetomidina por via intramuscular de forma isolada ou associada com acepromazina, sem ultrapassar, entretanto, os limites considerados insuficientes para a espécie.

Foi observado que os midriáticos em associação com dexmedetomidina podem ser necessários para facilitar os procedimentos oftálmicos que exigem dilatação da pupila (Artigas, Redondo \& Lopes-Murcia, 2012).

Rauser, Mrazova \& Zapletalova (2016) verificaram que o uso de dexmedetomidina ou medetomidina, por via intramuscular, associados com propofol e isoflurano resultou em miose, no entanto, sem uma redução significativa na pressão intraocular ou pressão arterial.

\section{Benzodiazepínicos}

Os benzodiazepínicos produzem bom relaxamento muscular e possuem ação anticonvulsivante através da ação 
inibitória do ácido gama aminobutírico (GABA), sendo geralmente associados à cetamina na medicação pré-anestésica, prevenindo os efeitos deletérios de contrações musculares e excitação (Zamorra, 1999). Podem ser utilizados com segurança na anestesia de pequenos animais para procedimentos oftálmicos, pois além de permitir um retorno suave da anestesia, promove um relaxamento dos músculos oculares (Bechara, 2002).

O diazepam administrado topicamente na conjuntiva reduz a PIO (Gross \& Pablo, 2015). Contudo, Kovalcuka e colaboradores (2013) afirmam que o diazepam sem associação eleva a PIO nos primeiros dez minutos após administração endovenosa, retornando à normalidade 20 minutos depois.

O midazolam associado ao butorfanol como pré-anestésicos foi capaz de reduzir aumentos da PIO induzidas pelo propofol na pesquisa realizada por Webb e colaboradores (2018), apesar do aumento da PIO ser transitório e ter sido verificado apenas no grupo de cães não glaucomatosos.

\section{Fenotiazinas}

O fenotiazínico mais utilizado na anestesia de cães e gatos é a acepromazina, um tranquilizante com propriedades antieméticas, que pode impedir a ocorrência de vômito e ânsia em pacientes com afecções oftálmicas submetidos à cirurgia intraocular ou que tenham o potencial de ruptura do bulbo ocular, devido sua ação na redução da PIO em até $20 \%$, tanto por via intravenosa quanto por via tópica em olhos que estejam com aumento da PIO, contudo esse efeito não foi observado em olhos com PIO normal. Além disso, quadros de êmese podem acarretar aumento rápido da PIO, podendo prejudicar o olho afetado, além do risco de romper a sutura (Tamura, 2003). Também foi observada diminuição da produção da lágrima com seu uso (McClure, Gelatt \& Gum, 1976).

A administração de acepromazina causou miose em cães ao ser utilizada de forma isolada ou em associação com tramadol, em estudo realizado por Santos e colaboradores (2013), além de diminuição na produção lacrimal, verificada por meio do teste lacrimal de Schirmer.

\section{Opióides}

Os analgesicos opióides são utilizados para produzir analgesia sem haver perda de consciência. Sua ação é mediada pelos receptores de opióides presentes principalmente na região cerebral e na parte dorsal da medula espinhal (Wagner, 2002). Eles são comumente usados em consonância com drogas tranquilizantes e outros analgésicos para melhorar a sedação, além de reduzir a quantidade necessária dos demais fármacos administrados na anestesia para se obter os efeitos desejados (Monteiro et al., 2008).

A morfina pode atuar na diminuição da PIO, contudo deve ser considerado que o uso sistêmico de alguns opióides poderá causar vômito e consequentemente a elevação da PIO devido à emese e atividade eferente vagal, que também poderá predispor o paciente ao ROC (Gross \& Giuliano, 2017).

Os opióides podem induzir a miose e impedem a midríase necessária para a cirurgia de catarata. Quando isso ocorrer, pode-se considerar a administração de naloxona (Kaswan, Quandt \& Morre, 1992; Stephen, Vestre \& Stiles, 2003).

O estroma superficial possui terminações nervosas (Slatter, 2005), portanto quadros de ceratite ulcerativa causam bastante algia ao paciente devido a estimulação dos mesmos, nesses casos a administração tópica de solução de sulfato de morfina a $1 \%$ proporciona analgesia local trazendo conforto ao paciente durante o tratamento (Stiles, Honda \& Krohne, 2003).

\section{Considerações Finais}

A elaboração de um protocolo anestésico para qualquer paciente envolve não apenas a escolha apropriada do agente anestésico, mas também um plano de conduta capaz de assegurar a qualidade do resultado pós-operatório. Isto requer 
conhecimento do estado físico do paciente e do procedimento oftálmico específico a ser realizado, além de familiaridade com a fisiologia e farmacologia ocular.

\section{Referências}

Aghababaei, A., Ronagh, A., Mosallanejad, B., \& Baniadam, A. (2021). Effects of Medetomidine, Dexmedetomidine and their combination with Acepromazine on the intraocular pressure (IOP), tear secretion and pupil diameter in dogs. Veterinary Medicine and Science 15.

Artigas, C., Redondo, J. I., Lopez-Murcia M. M. (2012). Effects of intravenous administration of dexmedetomidine on intraocular pressure and pupil size in clinically normal dogs. Vet Ophthalmol. 15, 79-82.

Batista C. M., Laus J. L., Nunes N. et al. (2000). Evaluation of intraocular and particial CO2 pressure in dogs anesthetized with propofol. Veterinary Ophthalmology. 3(1):pp.17-19.

Bechara, J. N. (2002). Anestesia em oftalmologia. In: Fantoni D.T \& Cortopassi S.R.G. Anestesia em cães e gatos. (pp.271-279). Roca.

Borgeat, A., Wilder-Smith, Oliver H. G., \& Suter, Peter M. (1994). The nonhypnotic therapeutic applications of propofol. American Society of Anesthesiologists. 80, 642-656.

Borges, A. C. do N., Costa, A. L., Bezerra, J. B., Araújo, D. S., Soares, M. A. A., Gonçalves, J. N. de A., Rodrigues, D. T. da S., Oliveira, E. H. S. de, Luz, L. E. da, Silva, T. R., \& Silva, L. G. de S. (2020). Epidemiologia e fisiopatologia da sepse: uma revisão. Research, Society and Development, 9(2), e187922112.

Burke, J. A., \& Potter, D. E. (1986). The ocular effects of xylazine in rabbits, cats, and monkeys. J Ocul Pharmacol. 2 , 9-21.

Calderone, L., Grimes, P., \& Shalev, M. (1986). Acute reversible cataract induced by xylazine and by ketamine-xylazine anesthesia in rats and mice. Exp Eye Res. 42, 331-337.

Clutton, R. E., Boyd, C., Richards, D. L. S., et al. (1988) Significance of the oculocardiac reflex during ophthalmic surgery in the dog. J Small Anim Pract. 29, 573-579.

Donlon, J. V. Jr. (1988). Anesthesia for ophthalmic surgery. In: Barash P, ed. ASA Refresher Course Lectures (vol 16. pp. 81-92). JB Lippincott

Esson, D. W. (2015). Normal Ocular Anatomy.Clinical Atlas of Canine and Feline Ophthalmic Disease, (pp.1-3) Saunders, Elsevier Inc.

Gross M. E., \& Pablo L. S., (2015). Ophthalmic patients. In: Grimm KA, Lamont LA, Tranquilli WJ. Lumb \& Jones' Veterinary Anesthesia and Analgesia. Ames: John Wiley \& Sons, (pp.963-982).

Gross, M. E., \& Giuliano, E. A. (2007). Anesthesia and analgesia for selected patients and procedures: ocular patients. In: Tranquilli WJ, Thurmon JC, Grimm KA, eds. Lumb and Jones' Veterinary Anesthesia and Analgesia ,(5th edn. 1056 páginas). Ames, IA: Blackwell Publishing.

Grubb, T., Sager, J., Gaynor, J. S., Montgomery, E., Parker, J. A., Shafford, H., \& Tearney, C. (2020). Anesthesia and Monitoring Guidelines for Dogs and Cats. Journal of the American Animal Hospital Association. 56, 59-82.

Hsu, W. H., Lee, P., \& Betts, D. M. (1981). Xylazine-induced mydriasis in rats and its antagonismo by $\alpha$-adrenergic blocking agents. $J$ Vet Pharmacol Ther. 4:.97-101.

Jia, L., Cepurna, W. O., Johnson, E. C., et al. (2000). Effect of general anesthetics on IOP in rats with experimental aqueous outflow obstruction. Invest Ophthalmol Vis Sci. 41: 3415-3419.

Jin, Y., Wilson, S., Elko, E. E., et al. (1991). Ocular hypotensive effects of medetomidine and its analogs. J Ocul Pharmacol. (7: $285-296$.

Kaswan, R. L., Quandt, J. E., \& Moore, P. A. (1992). Narcotics, miosis, and cataract surgery [letter]. J Am Vet Med Assoc. 201:1819-1820.

Koche, J.C. (2011). Fundamentos de metodologia científica: Teoria da ciência e iniciação a pesquisa. Editora Vozes, 185p.

Kovalcuka L, Birgele E, Bandere D. et al. (2013). The effects of ketamine hydrochloride and diapezam on the intraocular pressure and pupil diameter of the dog's eye. Veterinary Ophthalmology. 16(1):29-34.

Krupin, T., Cross, D. A., Becker, B. (1997). Decreased basal tear production associated with general anesthesia. (95:pp.107-108). Arch Ophthalmol.

Madruga, G. M., Ruiz, T., Ribeiro, A. P. (2015). Efeitos dos anestésicos na pressão intraocular em cães e gatos. Investigação. 14(2):28-32.

Maggs, D. J. (2013). Cornea and Sclera. In D. J. Maggs, P. E. Miller, \& R. Ofri (Eds.), Slatter's Fundamentals of Veterinary Ophthalmology. 5.184-219. Saunders, Elsevier Inc.

McClure, J. R., Gelatt, K. N., Gum, G. G., et al. (1976). The effect of parenteral acepromazine and xylazine on intraocular pressure in the horse. Vet Med Small Anim Clin.71: 1727-1730.

Miller, P. E. (2013). Lacrimal System. In D. J. Maggs, P. E. Miller, \& R. Ofri (Eds.), Slatter's Fundamentals of Veterinary Ophthalmology. 5. 165-183. Saunders, Elsevier Inc.

Murphy, C. J., Samuelson, D. A., Pollock, R. V. H. (2012). Ch. 21: The Eye. Miller's Anatomy of the Dog (pp.746-785). 
Ofri, R. (2013). Retina. In D. J. Maggs, P. E. Miller, \& R. Ofri (Eds.), Slatter's Fundamentals of Veterinary Ophthalmology. (5, 299-333. Saunders, Elsevier Inc.

Ofri, R. (2013). Vitreous. In D. J. Maggs, P. E. Miller, \& R. Ofri (Eds, 6). Slatter's Fundamentals of Veterinary Ophthalmology. (291-298). Saunders, Elsevier Inc.

Ofri, R. (2013a). Neuroophthalmology. In D. J. Maggs, P. E. Miller, \& R. Ofri (Eds.), Slatter's Fundamentals of Veterinary Ophthalmology. 334-371.

Pinto, R. B. B., Ribeiro, K. C., Silva, M. F. da, Regalin, D., Meirelles-Bartoli, R. B., \& Amaral, A. V. C. do. (2021). Principais bloqueios anestésicos para cirurgias oculares em cães e gatos. Research, Society and Development, 10(3), e55210313719. https://doi.org/10.33448/rsd-v10i3.13719

Pontes, K. C. S. et al. (2010). A comparison of the effects of propofol and thiopental on tear production in dogs. Revista Ceres. 57(6), 757-761.

Potter, D. E., Ogidigben, M. J. (1991). Medetomidine-induced alterations of intraocular pressure and contraction of the nictitating membrane. Invest Ophthalmol Vis Sci. 32, 2799-2805.

Rauser P., Mrazova M., \& Zapletalova J. (2016). Influence of dexmedetomidine-propofol-isoflurane and medetomidine-propofol-isoflurane on intraocular pressure and pupil size in healthy dogs. Veterinarni Medicina. 61: 635-642.

Ribeiro, L. M., Ferreira, D. A., Brás, S., Castro, A., Nunes, C. A., Amorim, P., \& Antunes, L. M. (2009). Correlation between clinical signs of depth of anaesthesia and cerebral state index responses in dogs during induction of anaesthesia with propofol. Research in veterinary science. 87(2) 287-291.

Samuelson, D. A. (2013). Ophthalmic anatomy. In: Gelatt, K. N.; Gilger, B. C.; Kern, T. J. (Ed.). Veterinary ophthalmology. (5a ed.), 39-170. Ames: WileyBlackwell.

Santos, P. H. A. et al. Comparação do diâmetro da pupila e produção de lágrimas em cães tratados com acepromazina, tramadol e suas combinações. 60(2) Revista Ceres.

Sharpe, L. G., Pickworth, W. B. (1985). Opposite pupillary size effects in the cat and dog after microinjections of morphine, normorphine and clonidine in the Edinger-Westphal nucleus. Brain Res Bull.15: 329-333.

Shepard, M. K., Accola, P. J., Lopez, L. A, et al. (2011). Effect of duration and type of anesthetic on tear production in dogs. Am J Vet Res. 72: 608-612.

Slatter, D. (2005). Fundamentos em Oftalmologia Veterinária. (3a ed.), 283- 338. Roca.

Stephen, D. D., Vestre, W. A., Stiles, J., et al. (2003). Changes in intraocular pressure and pupil size following intramuscular administration of hydromorphone hydrochloride and acepromazine in clinically normal dogs. Vet Ophthalmol.6:.73-76.

Stiles, J., Honda, C. N., Krohne, S. G., et al. (2003). Effect of topical administration of $1 \%$ morphine sulfate solution on signs of pain and corneal wound healing in dogs. Am J Vet Res. 64: 813-818.

Varela F. E., \& Mendoza X.P. (2007). Influencia del propofol y tiopental en la presión intraocular durante la inducción de la anestesia. Revista Médica de los Post Grados de Medicina. 10(1).

Verbruggen, A. M., Akkerdaas, L. C., Hellebrekers, L. J., \& Stades, F. C. (2000). The effect of intravenous medetomidine on pupil size and intraocular pressure in normotensive dogs. The veterinary quarterly. 22(3), 179-180.

Wagner, A. E. (2002). Opioids In: Handbook Veterinary Pain Management. Gaynor, J. S.; Muir, W. W. (pp.164-183).

Webb, T. R., Wyman, M., Smith, J. A., Ueyama, Y., \& Muir, W. W. (2018). Effects of propofol on intraocular pressure in premedicated and nonpremedicated dogs with and without glaucoma. Journal of the American Veterinary Medical Association. 252(7): 823-829.

Zamorra, V. G. (1999). Protocolo preanestesico y anestésico utilizado em la clínica de pequeños animales de Universidad Nacional de Colombia em pacientes caninos y felinos. Revista de Medicina Veterinaria y Zootecnia. 25-29. 